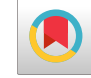

\title{
Investigation of Possible Role of Chlamydia in Pseudoexfoliation Syndrome
}

\author{
Serpil Gonca ${ }^{1,{ }^{*}}$, Mehmet Sami Serin (iD ${ }^{1}$, Ayça Yılmaz ${ }^{2}$, Özgün Oktay ${ }^{2}$, Ayșe Serin ${ }^{3}$ and Zehra Öksüz ${ }^{1}$ \\ ${ }^{1}$ Department of Pharmaceutic Microbiology, Faculty of Pharmacy, Mersin University, Mersin, Turkey \\ ${ }^{2}$ Department of Ophthalmology, Faculty of Medicine, Mersin University, Mersin, Turkey \\ ${ }^{3}$ Department of Forensic Science, Faculty of Medicine, University of Çukurova, Adana, Turkey \\ "Corresponding author: Department of Pharmaceutic Microbiology, Faculty of Pharmacy, Mersin University, Mersin, Turkey. Email: serpilgonca@mersin.edu.tr
}

Received 2021 June 16; Revised 2021 July 22; Accepted 2021 July 24.

\begin{abstract}
Background: Pseudoexfoliation syndrome (PES) is a systemic disease characterized by the aggregation of fibrillar extracellular material in intraocular and extraocular tissues with unknown etiology. Clarifying the etiopathogenesis of PES would be important for public health.

Objectives: We aimed to investigate the possible role of Chlamydia in the etiology of PES.

Methods: This cross-sectional study was carried out in the ophthalmology clinic of a tertiary hospital. The study included two groups, including the patient group (PES patients with nuclear cataracts) and the control group (patients with nuclear cataracts). Patients with other ophthalmic problems and systemic diseases were excluded. Blood samples and conjunctival swabs taken from 49 patients and 42 controls were used in the study. Anti-Chlamydia trachomatis IgG and IgM, anti-C. pneumoniae IgG and IgM, Interleukin (IL)-6, and IL-20 were studied in the serum samples. The PCR study was performed with conjunctival swab samples and sequence analysis of PCR-positive samples was performed.

Results: According to the results of the study, there was no statistically significant difference between the groups in terms of anti-C. trachmatis IgG, anti-C. trachmatis IgM, anti-C. pneumoniae IgM, IL-6, and PCR results. There was a statistically significant difference between patient and control groups in terms of anti-C. pneumoniae IgG and IL-20 levels. The DNA sequencing of all PCR products was found to be compatible with C. pneumoniae.

Conclusions: It seems that C. pneumoniae might have an important role in the etiology and development of PES. However, further studies in larger groups are needed to clarify these results.
\end{abstract}

Keywords: Pseudoexfoliation Syndrome, Chlamydia, Interleukin-6, Interleukin-20

\section{Background}

Chlamydiaceae family contains 11 species that are pathogenic for animals or humans (1). Chlamydia trachomatis and $C$. pneumoniae are common human pathogens responsible for a broad range of illnesses $(1,2)$. Strains of C. trachomatis are separated into three biovars, and they are further subtyped by serovars. In developing countries, trachoma biovars (A-C) are the leading causes of non-congenital blindness (3). Chlamydia pneumoniae is a common infectious agent in the world that causes respiratory tract infections, accounting for approximately $10 \%$ of community-acquired pneumonia. In addition, the symptoms of $C$. pneumoniae infection range from asymptomatic to pneumonia and also cause various chronic diseases such as atherosclerosis, asthma, and arthritis $(3,4)$. Moreover, it has been reported that C. pneumoniae may be linked to age-related macular degeneration (5). This pathogen interacts with different cell types such as epithelial cells, monocytes/macrophages, smooth muscle cells, and endothelial cells (4).

Chlamydial antigens are recognized through a variety of receptors, e.g., cell surface receptors, cytosolic innate immune sensors, and endosomal receptors (3). It is known that the activation of these receptors induces a strong chemokine and cytokine response that enhances PMN infiltration into the infected field. In this way, in advance entry into a new cell, EBs are subjected to PMNs, and their function is to clear the infection (6). However, this inflammatory reaction, which is necessary for bacterial clearance, also becomes a reason for immunopathology such as scarring and tissue damage (3).

Pseudoexfoliation syndrome (PES) is a systemic disease characterized by the aggregation of fibrillar extra- 
cellular material in intraocular and extraocular tissues (7, $8)$. Due to the aggregation of pseudoexfoliation material in the eye, different complications such as weak pupillary dilation, cataract formation, pseudoexfoliative glaucoma (PEG), pseudouveitis, posterior synechia, and zonular weakness may occur (9). Jammal et al. studied the characteristics of patients with PES. They reported that PEG was detected at $25.4 \%$ in patients with PES (10). The presence of fibrillary material may enhance the risk of complications after and during cataract surgery and of late dislocation of intraocular lenses (8).

Joshi and Singanwad (11) studied surgical difficulties linked with PES and noticed that intraoperative difficulties were present in $27.4 \%$ of PES patients. The presence of fibrillar material in extraocular tissues such as the heart, lung, kidneys, liver, and meninges has been demonstrated via electron/light microscopes (12). Pseudoexfoliation syndrome is generally seen in older age groups and its prevalence varies according to race, geographic region, and ethnicity (8). The factors leading to the development of PES are not yet fully understood (12). There are various studies showing the association of PES with systemic diseases such as vascular (13), cardiovascular (14), and cerebrovascular diseases (15). In addition, PES involves low-grade inflammation (16).

Cytokines are protein mediators, which participate in many physiological processes and play a key role in inflammation. Increased production of pro-inflammatory cytokines is related to disease progression (17). Inflammatory molecules in patients with PES, such as IL-6, C-reactive protein, plasma homocysteine, and IL-1 $\beta$ were detected at higher levels (9). Also, there are studies showing an increase in IL-6 levels in Chlamydia infections and patients with PES $(5,18)$. In addition, IL6 is a pivotal cytokine in the integrated immune response and has been targeted in the treatment of numerous inflammatory diseases (19). Besides, IL-6 is used as a biomarker because of its central role in inducing and maintaining the inflammatory response (20). Also, IL-20 is a member of the IL-10 family. These cytokines are thought to be associated with the pathogenesis of autoimmune and chronic inflammatory diseases such as inflammatory bowel disease, psoriasis, and rheumatoid arthritis (21). In this study, IL-20 was chosen as another marker of inflammation since we did not observe a study investigating IL-20 in patients with PES in the literature screening.

In several studies on PES, it has been observed that PES is associated with various systemic disorders, but none has been carried out on Chlamydia associations of the disor$\operatorname{der}(9,12,22,23)$. Inflammation lays behind the common pathogenesis of these diseases. Therefore, we hypothesized that infection with Chlamydia might be an additional risk factor for the etiopathogenesis of PES-related diseases. In the present study, IgG and IgM antibodies against C. trachomatis and C. pneumoniae along with IL-6 and IL-20 were investigated in the blood samples taken from patient and control groups. Also, the C. trachomatis and C. pneumoniae 16S rRNA regions were amplified with the PCR method using conjunctival swab samples taken by viral transport.

\section{Objectives}

In the present study, we aimed to investigate the possible role of Chlamydia in the etiology of PES.

\section{Methods}

\subsection{Study Group}

The study group comprised 49 PES patients with nuclear cataracts, and the control group comprised 42 patients with nuclear cataracts. Patients with ophthalmic diseases such as primary open-angle glaucoma, uveitis, and age-related macular disease were not involved in the study or control group. Having systemic diseases, such as hypertension, diabetes, or malignancies were the other exclusion criteria. The control group consisted of individuals who did not have any systemic and ophthalmic diseases other than cataracts. Peripheral blood samples and conjunctival swab samples were taken from the patients. The patient group included 22 females and 27 males $(n=49$, age range 53 - 88 years). The control group included $15 \mathrm{fe}$ males and 27 males ( $n=42$, age range $53-84$ years). Peripheral blood samples $(5 \mathrm{~mL})$ from the patients were centrifuged at $5000 \times \mathrm{g}$ for five minutes. Then, serum samples were stored at $-80^{\circ} \mathrm{C}$ until analysis. Conjunctival swab samples that were taken with the universal transport medium (UTM-RT; Copan, Italy) were also stored at $-80^{\circ} \mathrm{C}$ until analysis.

\subsection{ELISA Assays}

The ELISA studies were performed on collected serum samples to detect antibodies against $C$. trachomatis and $C$. pneumoniae and to determine IL-6 and IL-20 cytokine levels. Serum samples were studied as recommended by the manufacturers (C. trachomatis and C. pneumoniae [Euroimmun, Luebeck Germany IgM and IgG], IL-6 [DIAsource, Louvainla-Neuve, Belgium], and IL-20 [Sunred, Shanghai, China]). Optical density was determined by measuring at $450 \mathrm{~nm}$ with a spectrophotometer (Thermo Scientific, Multiskan Go Type 1510). 


\subsection{Nucleic Acid Extraction and PCR Assay}

Gene jet kit (GeneJET, Thermo Scientific, USA) was used for DNA extraction from conjunctival swab specimens. The DNA region of approximately $207 \mathrm{bp}-215$ bp (the length was variable according to the species) in the 16S rRNA genome was amplified by the PCR technique. Sequences of primers used for target gene region amplification panCh16F2 (5'-CCGCCAACACTGGGACT3') and panCh16R2 (5' -GGAGTTAGCCGGTGCTTCTTTAC-3') (24). The PCR products ( $3 \mu \mathrm{L}$ ) were resolved by agarose gel $(1 \%, \mathrm{w} / \mathrm{v})$ electrophoresis in $0.5 \mathrm{X}$ TBE buffer at $200 \mathrm{~V}$ for 30 $\mathrm{min}$. The DNA was visualized by UV illumination after staining with ethidium bromide. Water was used as a negative PCR control.

\subsection{DNA Sequencing and Comparative Analysis}

Amplicons of positive samples were purified using a GeneJet PCR Purification Kit (Thermo Scientific, USA). A sequencing PCR was performed with the specifically designed inner primers panFseq (5-CCAACACTGGGACTGAGA3) and panRseq (5-GCCGGTGCTTCTTTAC-3). The sequencing PCR assay was done using a BigDye Terminator V3.1 cycle sequencing kit (Applied Biosystems). Then, sequencing was performed in ABI PRISM 3130 Genetic Analyzer (Applied Biosystem, CA, USA). The obtained sequences were compared with the reference sequences containing 16S rRNA gene sequences of $C$. pneumoniae and C. trachomatis available from the GenBank Database. The GenBank accession numbers of reference sequences were L06108.1 (Chlamydia pneumoniae 16S ribosomal RNA sequence)(25), DQ019305.1 (Chlamydia trachomatis strain Ia/870 $16 \mathrm{~S}$ ribosomal RNA gene, partial sequence) (26), and DQ019306.1 (Chlamydia trachomatis strain J/UW36 16S ribosomal RNA gene partial sequence) (27). Nucleotide and amino acid alignment comparisons were carried out with the Clustal W method using the MEGA 7.0.21 program. Phylogenetic analyses were performed with the Neighbor-Joining (NJ) method using MEGA7.021.

\subsection{Statistical Analysis}

Positive and negative values obtained by ELISA and PCR studies in patient and control group samples were compared with the chi-square test for each group. Values of $P$ $<0.05$ were considered to indicate statistically significant differences. Comparisons were made with the GraphPad Prism 6 for Windows (version 6.01) program.

\section{Results}

A total of 91 participants ( 54 females and 37 males) were enrolled, including 49 PES patients with nuclear cataracts and 42 control patients with nuclear cataracts. The mean ages of the patient group and the control group were determined as 70.02 and 66.28 , respectively. In addition, 27 $(55.102 \%)$ of the patient group were males, 22 (44.897\%) were females, $27(64.285 \%)$ of the control group were males, and $15(35.714 \%)$ of the control group were females. Anti-C. trachomatis IgG/IgM and anti-C. pneumoniae IgG/IgM ELISA results and $P$ values are shown in Tables 1 and 2. According to the results, no significant difference was found between the patient and controls groups in anti-C. trachomatis IgG and IgM (P-values 0.0515 and 0.3253, respectively) (Table 1). While a statistically significant difference was detected in anti-C. pneumoniae IgG, no significant difference was detected in anti-C. pneumoniae IgM (P-values 0.0349 and 0.3882 , respectively) (Table 2 ).

Besides, IL-6 6 and IL-20 levels were studied in blood samples taken from the patient and control groups, and the results are shown in Table 3. As seen, while there was no statistically significant difference between the patient and control groups in IL6, a significant difference was found in IL20 levels (P-values 0.0612 and 0.0459 , respectively). The PCR results of conjunctival swab samples taken from the patient and control groups are shown in Table 4 and Figure 1. No statistically significant difference was found between the patient and control groups in Chlamydia PCR results (P-value 0.0928). Based on phylogenetic analysis, it was determined that all of the PCR-positive samples in the patient and control groups showed close homology to the $16 \mathrm{~S}$ rRNA gene sequence of C. pneumoniae although H30, $\mathrm{H} 42$, and K10 exhibited closer homology to it (Figure 2). The ELISA results of Chlamydia PCR-positive samples are shown in Table 5.

\section{Discussion}

Chlamydia trachomatis is a significant human pathogen and the cause of sexually transmitted infection and blinding trachoma (28). Trachoma is the leading contagious cause of blindness worldwide (29). The infection evokes an intensive mixed inflammatory response in the conjunctiva launched by cytokines and interferons delivered by infected cells (30). In the study, the presence of anti-C. trachomatis IgG and anti-C. trachomatis IgM was investigated in serum samples to investigate the presence of $C$. trachomatis antibodies in the patient and control groups. Anti-C. trachomatis IgG was detected in $6.12 \%$ of the patient group, while all of them were negative in the control group. Anti-C trachomatis IgM was detected as $4.08 \%$ in the patient group and $2.04 \%$ in the control group (Table 1). The low level of antibody positivity is thought to be the result of the availability of antibiotics and improvement in living and socio-economic conditions; besides, the introduction 


\begin{tabular}{|c|c|c|c|c|c|c|}
\hline & \multicolumn{2}{|c|}{ Chlamydia trachomatis IgG } & \multirow{2}{*}{$\mathbf{P}$} & \multicolumn{2}{|c|}{ Chlamydia trachomatis IgM } & \multirow{2}{*}{$\mathbf{P}$} \\
\hline & Patient Group No. (\%) & Control Group No. (\%) & & Patient Group, No. (\%) & Control Group No. (\%) & \\
\hline Positive & $3(6.12)$ & $0(0)$ & \multirow{3}{*}{0.0515} & $2(4.08)$ & $1(2.04)$ & \multirow{3}{*}{0.3253} \\
\hline Negative & $46(93.87)$ & $42(100.0)$ & & $47(95.91)$ & $41(97.61)$ & \\
\hline Total & $49(100.0)$ & $42(100.0)$ & & $49(100.0)$ & $42(100.0)$ & \\
\hline
\end{tabular}

Table 2. Anti-Chlamydia pneumoniae IgG and IgM Results in Case and Control Groups Measured by ELISA Technique

\begin{tabular}{|c|c|c|c|c|c|c|}
\hline & \multicolumn{2}{|c|}{ Chlamydia pneumoniae IgG } & \multirow{2}{*}{$\mathbf{P}$} & \multicolumn{2}{|c|}{ Chlamydia pneumoniae IgM } & \multirow{2}{*}{$\mathbf{P}$} \\
\hline & Patient Group, No. (\%) & Control Group, No. (\%) & & Patient Group, No. (\%) & Control Group, No. (\%) & \\
\hline Positive & $28(57.14)$ & $16(38.09)$ & \multirow{3}{*}{0.0349} & $3(6.12)$ & $2(4.76)$ & \multirow{3}{*}{0.3882} \\
\hline Negative & $21(42.85)$ & $26(61.90)$ & & $46(93.87)$ & $40(95.23)$ & \\
\hline Total & $49(100.0)$ & $42(100.0)$ & & $49(100.0)$ & $42(100.0)$ & \\
\hline
\end{tabular}

Table 3. IL-20 and IL-6 Cytokine Levels in Case and Control Groups Measured by ELISA Technique

\begin{tabular}{|c|c|c|c|c|c|c|}
\hline & \multicolumn{2}{|c|}{ IL-6 } & \multirow{2}{*}{$\mathbf{P}$} & \multicolumn{2}{|c|}{ IL-20 } & \multirow{2}{*}{$\mathbf{P}$} \\
\hline & Patient Group, No. (\%) & Control Group, No. (\%) & & Patient Group, No. (\%) & Control Group, No. (\%) & \\
\hline Positive & $0(0.0)$ & $2(4.76)$ & \multirow{3}{*}{0.0612} & $29(59.18)$ & $17(40.47)$ & \multirow{3}{*}{0.0459} \\
\hline Negative & $49(100.0)$ & $40(95.23)$ & & $20(40.81)$ & $25(59.52)$ & \\
\hline Total & $49(100.0)$ & $42(100.0)$ & & $49(100.0)$ & $42(100.0)$ & \\
\hline
\end{tabular}

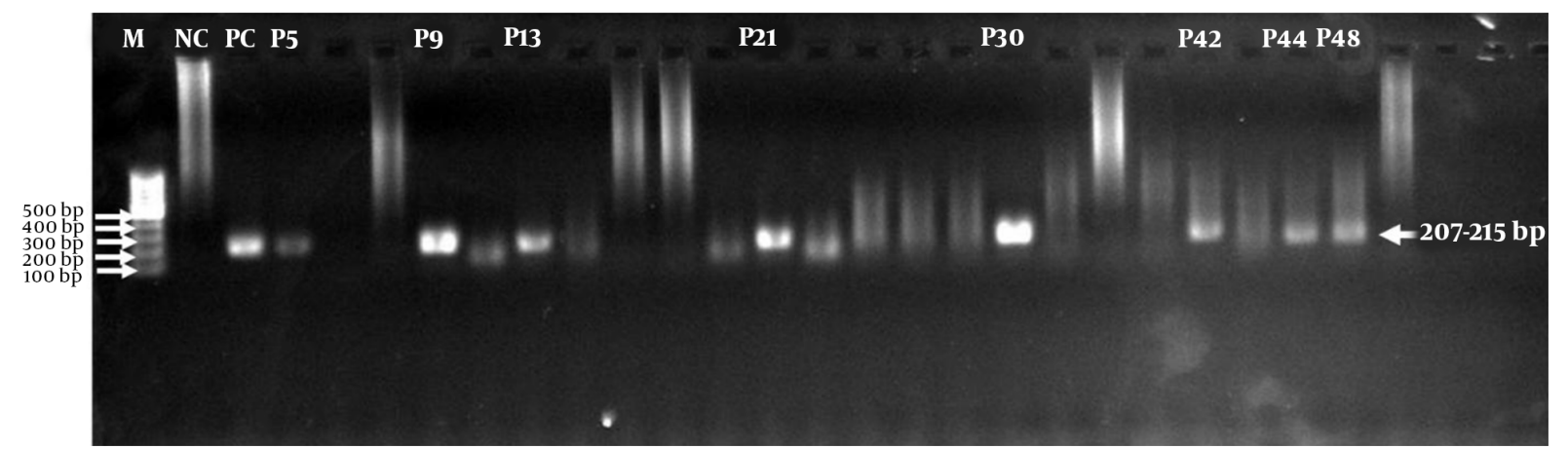

Figure 1. PCR results belonging to the patient group. Marker (M); Negative control (NC); Positive control (PC); P, patient sample.

Table 4. PCR Results of Conjunctival Swab Samples Taken from Patient and Control Groups

\begin{tabular}{l|c|c|c}
\hline & Patient Group, No. (\%) & Control Group, No. (\%) & P \\
\hline Positive & $8(16.32)$ & $3(7.14)$ & \\
\cline { 1 - 3 } Negative & $41(83.67)$ & $39(92.85)$ & \multirow{2}{*}{0.0928} \\
\cline { 1 - 3 } Total & $49(100.0)$ & $42(100.0)$ & \\
\hline
\end{tabular}

to National Trachoma Control programmes decreased the prevalence in developed countries. In addition, the introduction of the Global Elimination of Trachoma 2020 pro- gram by the WHO using SAFE (S, surgery to trachomatous trichiasis; A, antibiotics to C. trachomatis; F, facial cleaning; and $\mathrm{E}$, environmental development) strategy greatly reduced the prevalence (29).

Chlamydia pneumoniae regulates host cell functions throughout infection. For example, C. pneumoniae stimulates proinflammatory cytokines such as IL-8, IL-1b, and TNF mediated by toll-like receptor-2 (TLR-2) and TLR4 with/without NLR (NOD-like receptor) pyrin domaincontaining protein 3 (NLRP3) inflammasome activation (4). The activation of cell-mediated immune responses is presumed to be significant for protective immunity 


\begin{tabular}{|c|c|c|c|c|c|}
\hline & \multirow{3}{*}{$\begin{array}{c}\text { Chlamydia pneumoniae PCR } \\
\text { Results }\end{array}$} & \multicolumn{4}{|c|}{ ELISA Results } \\
\hline & & \multicolumn{4}{|c|}{ Anti-Chlamydia pneumoniae } \\
\hline & & IgG & IgM & IL-6 & IL-20 \\
\hline Sample P5 & $\mathrm{P}$ & $\mathrm{N}$ & $\mathrm{N}$ & $\mathrm{N}$ & $\mathrm{P}$ \\
\hline Sample P9 & $\mathrm{P}$ & $\mathrm{N}$ & $\mathrm{N}$ & $\mathrm{N}$ & $\mathrm{P}$ \\
\hline Sample P13 & $\mathrm{P}$ & $\mathrm{N}$ & $\mathrm{P}$ & $\mathrm{N}$ & $\mathrm{N}$ \\
\hline Sample P21 & $\mathrm{P}$ & $\mathrm{P}$ & $\mathrm{N}$ & $\mathrm{N}$ & $\mathrm{P}$ \\
\hline Sample P30 & $\mathrm{P}$ & $\mathrm{P}$ & $\mathrm{N}$ & $\mathrm{N}$ & $\mathrm{P}$ \\
\hline Sample P42 & $\mathrm{P}$ & $\mathrm{P}$ & $\mathrm{N}$ & $\mathrm{N}$ & $\mathrm{P}$ \\
\hline Sample P44 & $\mathrm{P}$ & $\mathrm{P}$ & $\mathrm{P}$ & $\mathrm{N}$ & $P$ \\
\hline Sample P48 & $\mathrm{P}$ & $\mathrm{P}$ & $\mathrm{P}$ & $\mathrm{N}$ & $\mathrm{P}$ \\
\hline Sample C3 & $\mathrm{P}$ & $\mathrm{P}$ & $\mathrm{N}$ & $\mathrm{P}$ & $\mathrm{P}$ \\
\hline Sample C10 & $\mathrm{P}$ & $\mathrm{N}$ & $\mathrm{N}$ & $\mathrm{N}$ & $\mathrm{P}$ \\
\hline Sample C14 & $\mathrm{P}$ & $\mathrm{P}$ & $\mathrm{N}$ & $\mathrm{N}$ & $\mathrm{P}$ \\
\hline
\end{tabular}

Abbreviations: N, negative; P, positive; Sample P, patient sample; Sample C, control sample.

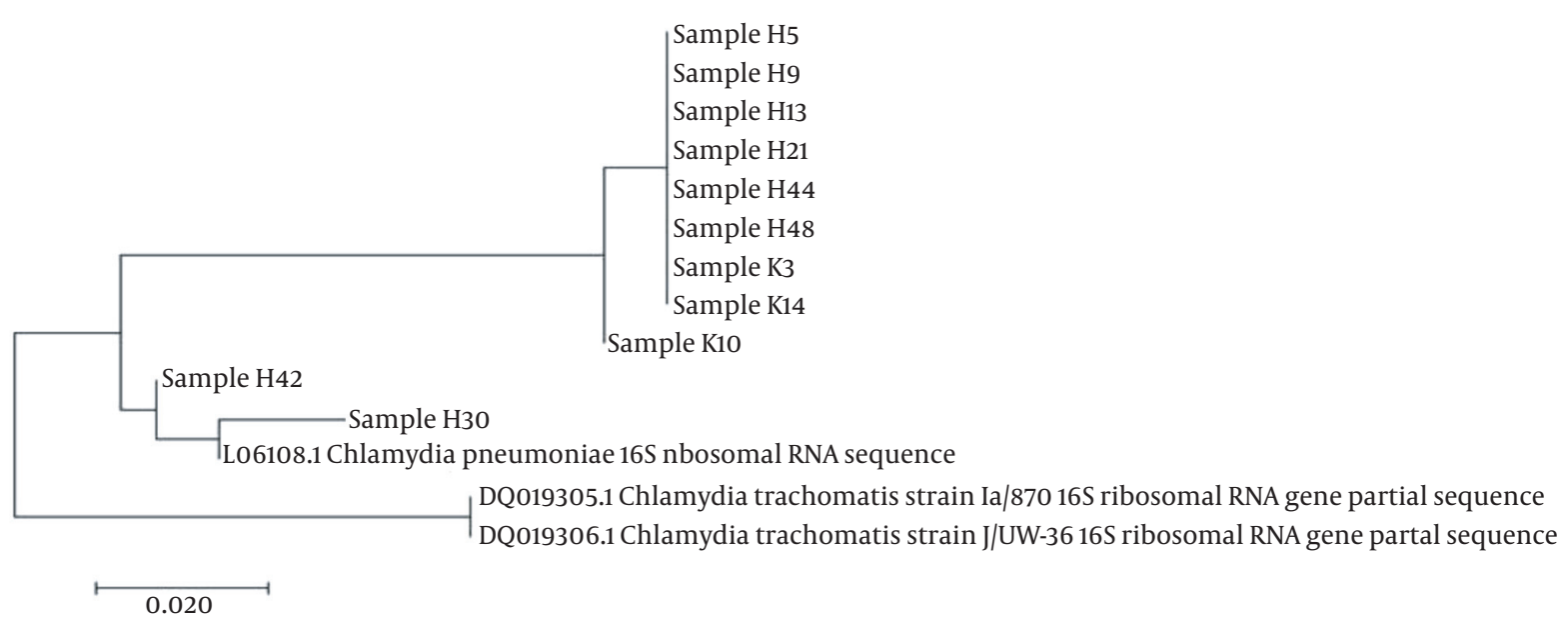

Figure 2. Comparative phylogenetic analysis of sequences obtained using sequence analysis with reference sequences. Sample H5, patient sample 5; Sample H9, patient sample 9; Sample H13, patient sample 13; Sample H21, patient sample 21; Sample H44, patient sample 44; Sample H48, patient sample 48; Sample K3, control sample 3; Sample K14, control sample 14; Sample K10, control sample 10; Sample H42, patient sample 42; Sample H30, patient sample 30; 10 L06108.1, Chlamydia pneumoniae 16S ribosomal RNA sequence; DQ019305.1, Chlamydia trachomatis strain Ia/870 16S ribosomal RNA gene, partial sequence; DQ019306.1, Chlamydia trachomatis strain J/UW36 16S ribosomal RNA gene partial sequence.

against this infection. The cytokine response of monocytes/macrophages contributes to two situations; it may contribute to infection control and induction of immune reactive disorders (31).

In the study, the presence of anti-C. pneumoniae IgG and IgM was studied in serum samples in order to investigate the presence of $C$. pneumoniae antibodies in the patient and control groups. Anti-C. pneumoniae IgG was detected in $57.14 \%$ of the patient group and $38.09 \%$ of the con- trol group. Anti-C. pneumoniae IgM was detected as $6.12 \%$ in the patient group and $4.76 \%$ in the control group (Table 2). According to the PCR results, $16.32 \%$ positivity in the patient group and $7.14 \%$ positivity in the control group was detected (Table 3 and Figure 1). Sequence analysis was done with the positive samples. Comparative phylogenetic analysis was performed using the sequences obtained after sequence analysis.

Based on phylogenetic analysis, it was determined that 
all of the PCR-positive samples in the patient and control groups showed close homology to the 16S rRNA gene sequence of C. pneumoniae although $\mathrm{H} 30, \mathrm{H} 42$, and $\mathrm{K} 10$ exhibited closer homology to it (Figure 2). No study examining the relationship between Chlamydia and PES pathogenesis was found in the literature review. However, there are several studies showing that $C$. pneumoniae is associated with various systemic diseases. Yuki et al. (32) noticed that higher $C$. pneumoniae titers are associated with POAG (primary open-angle glaucoma). In another study, Miao et al. (33) showed that TLR2/CXCR4 co-association makes easier C. pneumoniae infection-stimulated speed-up of atherosclerosis. The fact that anti-C. pneumoniae antibody rates were higher in the patient group than in the control and the PCR results obtained from conjunctival swab samples were compatible with C. pneumoniae suggests that these findings are important data that should be supported by further studies.

In addition, IL-6 and IL-20, which are the markers of inflammation, were studied in the patient and control groups. According to IL-6 ELISA results, no positivity was detected in the patient group, while it was $4.76 \%$ in the control group (Table 3). Chlamydia trachomatis infection stimulates the overproduction of a diversity of inflammatory cytokines such as IL-8, IL-6, TNF, granulocytemacrophage colony-stimulating factor, growth-regulated oncogene-alpha, and chemokines, which are involved in Chlamydial immunopathology. Throughout acute infection, C. trachomatis stimulates these inflammatory mediators to reduce the host immune response; as the repeated or prolonged infection happens, more immune cells and inflammatory mediators are delivered to fighting the infection. The overproduction of inflammatory mediators contributes notably to disease symptoms by damaging neighboring cells. Such investigations confirm a close relationship between the host immune system and Chlamydia (34). Chronic infectious agents such as C.pneumoniae cause an increase in inflammatory markers such as C-reactive protein, leukocyte, TNF, IL-1, and IL-6, which are known to be associated with atherosclerosis (35).

Moreover, there are studies examining the role of cytokines in the PES group. Sarenac Vulovic et al. (36) examined the role of proinflammatory cytokines (IL-6, TNF$\alpha$, and IL-17) in the PES formation process in the serum. They found that IL-6 levels were highly significant in the PES group compared to the control and PEG groups. No difference was detected in TNF, whereas IL-17 was not detected (36). Yildirim et al. (18) studied the role of cytokines in PES and noticed that the significant result was related to the mean IL-6 levels that were higher in the PES group than in the control group. In another study, Takai et al. (37) studied multiplex cytokines in the aqueous humor and showed that except for a significant reduction in IL-6 in the POAG group, the other cytokine levels were not different in the POAG and PEG groups compared to the cataract group. But, in our study, IL- 6 was found to be negative in the patient group. This situation can be explained by the work of Zenkel et al. (38), who indicated that the levels of interleukins IL-6 and IL-8 in the aqueous humor were three folds higher than that in controls in the early stage of the disease but not in the late stage or XFG.

Another inflammation marker, IL-20, was detected in $59.18 \%$ of the patient group and $40.47 \%$ of the control group (Table 3 ). As known, IL-20 is an IL-10 family member cytokine. Members of the IL-10 family of cytokines play important roles in regulating immune responses during host defense, as well as in autoimmune disorders, inflammatory diseases, and cancer (39). It is also known that these cytokines provoke the innate immune response to limit tissue epithelial damage resulting from viral and bacterial infections (40). No study investigating IL-20 levels in patients with PES was found in the literature review. However, the increased specific antibody against $C$. pneumoniae in PES patients in comparison with controls suggests a possible association between PES and C. pneumoniae infection.

As mentioned earlier, C. pneumoniae is also found in atherosclerotic lesions (3). Thus, macrophages infected with C. pneumoniae may enter the circulatory system and spread to various organs. Later, macrophages infected with C.pneumoniae up-regulate the expression of adhesion molecules and stimulate the production of inflammatory cytokines $(5,35)$. As known, studies have shown that IL-20 is released in eye injuries (41). However, our findings suggest that it may be caused by possible autoimmune disorders, inflammatory diseases, and tissue disorders due to viral and/or bacterial diseases in both PES and control groups. The ELISA results of the PCR-positive samples are given in Table 5. Accordingly, anti-C. pneumoniae IgG, antiC. pneumoniae IgM, IL-6, and IL-20 positivity percentages were $63.63 \%$, 27.27\%, 9.09\%, and 90.90\%, respectively. Based on the results of comparing the blood and conjunctiva swab samples of PCR-positive patients, we can interpret that $C$. pneumoniae may have a role in the etiology and pathogenesis of PES. However, this hypothesis needs to be investigated with further analysis and a wider patient population.

\subsection{Conclusions}

It is important for public health to elucidate the factors that may cause PES because PEG is observed at a higher rate in patients with PES and also because of the complications of cataract surgery in these patients. According to the literature review, no study has investigated the link between C. pneumoniae and PES. The relationship between 
this pathogen and PES should be further investigated because this would be a new approach to use antibiotics to treat vision-threatening optic neuropathy (pseudoexfoliation glaucoma). This would be an important guide for ophthalmologists. Moreover, it would be possible to obtain findings that significantly minimize unnecessary medication use and thereby time loss, side effects, and economic loss. Thus, our findings provide valuable contributions to this field in the literature. Although C. pneumoniae may have an important role in the etiology and development of PES, further studies are needed to clarify these results. In addition, there is a need for future large-scale studies to elucidate the associations and systemic features of PES syndrome.

\section{Footnotes}

Authors' Contribution: Study concept and design: MSS, AY, and SG. Acquisition of data: MSS, AY, SG, OO, and AS. Analysis and interpretation of data: SG, ZO, MSS, AY, AS, and OO. Drafting of the manuscript: SG, ZO, MSS, AY, AS, and OO. Critical revision of the manuscript for important intellectual content: SG, ZO, MSS, AY, AS, and OO. Statistical analysis: MSS.

Conflict of Interests: The authors declare that they have no conflict of interest.

Ethical Approval: The study was performed as per the Declaration of Helsinki and was approved by the Ethics Committee of the Mersin University Clinical Research (date: 02/11/2016; number: 2016/341).

Funding/Support: This study was supported by the Mersin University Scientific Research Projects (BAP) under Grant [ 2016-2-TP3-1827].

Informed Consent: Written informed consent was obtained from the patients before sample collection and after explaining the objectives and purpose of the study to them.

\section{References}

1. Gitsels A, Van Lent S, Sanders N, Vanrompay D. Chlamydia: what is on the outside does matter. Crit Rev Microbiol. 2020;46(1):100-19. doi: 10.1080/1040841X.2020.1730300. [PubMed: 32093536].

2. Desai $M$, Zhang H, Fan H. Optimal cultivation of Chlamydia requires testing of serum on individual species. BMC Res Notes. 2020;13(1). doi: 10.1186/s13104-020-4893-9.

3. Elwell C, Mirrashidi K, Engel J. Chlamydia cell biology and pathogenesis. Nat Rev Microbiol. 2016;14(6):385-400. doi: 10.1038/nrmicro.2016.30. [PubMed: 27108705]. [PubMed Central: PMC4886739].

4. Matsuo J, Sakai K, Okubo T, Yamaguchi H. Chlamydia pneumoniae enhances Interleukin 8 (IL-8) production with reduced azithromycin sensitivity under hypoxia. APMIS. 2019;127(3):131-8. doi: 10.1111/apm.12924. [PubMed: 30746791].
5. Ishida O, Oku H, Ikeda T, Nishimura M, Kawagoe K, Nakamura $\mathrm{K}$. Is Chlamydia pneumoniae infection a risk factor for age related macular degeneration? Br J Ophthalmol. 2003;87(5):523-4. doi: 10.1136/bjo.87.5.523. [PubMed: 12714382]. [PubMed Central: PMC1771658].

6. Rajeeve K, Das S, Prusty BK, Rudel T. Chlamydia trachomatis paralyses neutrophils to evade the host innate immune response. Nat Microbiol. 2018;3(7):824-35. doi: 10.1038/s41564-018-0182-y. [PubMed: 29946164].

7. Cicek Yilmaz D, Yilmaz A, Goksel I, Cirit A, Sen F, Tamer L. Evaluation of left ventricular functions in patients with pseudoexfoliation syndrome using tissue Doppler echocardiography and its association with plasma BNP levels. Anadolu Kardiyol Derg. 2014;14(5):422-6. doi: 10.5152/akd.2014.4878. [PubMed: 24901019].

8. Vardhan SA, Haripriya A, Ratukondla B, Ramulu P, Shivakumar C, Nath $\mathrm{M}$, et al. Association of pseudoexfoliation with systemic vascular diseases in a south Indian population.JAMA Ophthalmol. 2017;135(4):34854. doi: 10.1001/jamaophthalmol.2017.0064. [PubMed: 28278318].

9. Okutucu M, Findik H, Aslan MG, Arpa M. Is Netrin-1 deficiency responsible for inflammation and systemic diseases related to pseudoexfoliation? J Glaucoma. 2020;29(11):1077-81. doi: 10.1097/IJG.0000000000001624. [PubMed: 32769729].

10. Jammal H, Abu Ameera M, Al Qudah N, Aldalaykeh M, Abukahel A, Al Amer A, et al. Characteristics of patients with pseudoexfoliation syndrome at a tertiary eye care center in Jordan: A retrospective chart review. Ophthalmol Ther. 2021;10(1):51-61. doi: 10.1007/s40123-020-00319w. [PubMed: 33123988]. [PubMed Central: PMC7886922].

11. Joshi RS, Singanwad SV. Frequency and surgical difficulties associated with pseudoexfoliation syndrome among Indian rural population scheduled for cataract surgery: Hospital-based data. Indian J Ophthalmol.2019;67(2):221-6.doi:10.4103/ijo.IJO_931_18. [PubMed:30672474]. [PubMed Central: PMC6376804].

12. Yildirim N, Yasar E, Gursoy H, Colak E. Prevalence of pseudoexfoliation syndrome and its association with ocular and systemic diseases in Eskisehir, Turkey. Int J Ophthalmol. 2017;10(1):128-34. doi: 10.18240/ijo.2017.01.21. [PubMed: 28149789]. [PubMed Central: PMC5225361].

13. Gonen KA, Gonen T, Gumus B. Renal artery stenosis and abdominal aorta aneurysm in patients with pseudoexfoliation syndrome. Eye (Lond). 2013;27(6):735-41. doi: 10.1038/eye.2013.56. [PubMed: 23579404]. [PubMed Central: PMC3682361].

14. Praveen MR, Shah SK, Vasavada AR, Diwan RP, Shah SM, Zumkhawala $\mathrm{BR}$, et al. Pseudoexfoliation as a risk factor for peripheral vascular disease: a case-control study. Eye (Lond). 2011;25(2):174-9. doi: 10.1038/eye.2010.175. [PubMed: 21127507]. [PubMed Central: PMC3169212].

15. Yuksel N, Anik Y, Altintas O, Onur I, Caglar Y, Demirci A. Magnetic resonance imaging of the brain in patients with pseudoexfoliation syndrome and glaucoma. Ophthalmologica. 2006;220(2):125-30. doi: 10.1159/000090578. [PubMed: 16491036].

16. Borras T. Growth factors, oxidative damage, and inflammation in exfoliation syndrome. J Glaucoma. 2018;27 Suppl 1:S54-60. doi: 10.1097/IJG.0000000000000904. [PubMed: 29401156]. [PubMed Central: PMC6028288].

17. Fatkhullina AR, Peshkova IO, Koltsova EK. The role of cytokines in the development of atherosclerosis. Biochemistry (Mosc). 2016;81(11):135870. doi: 10.1134/S0006297916110134. [PubMed: 27914461]. [PubMed Central: PMC5471837].

18. Yildirim Z, Yildirim F, Ucgun NI, Sepici-Dincel A. The role of the cytokines in the pathogenesis of pseudoexfoliation syndrome. Int J Ophthalmol. 2013;6(1):50-3. doi: 10.3980/j.issn.2222-3959.2013.01.10. [PubMed: 23550261]. [PubMed Central: PMC3580249].

19. Rose-John S, Winthrop K, Calabrese L. The role of IL-6 in host defence against infections: immunobiology and clinical implications. Nat Rev Rheumatol. 2017;13(7):399-409. doi: 10.1038/nrrheum.2017.83. [PubMed: 28615731]. 
20. Kany S, Vollrath JT, Relja B. Cytokines in inflammatory disease. Int J Mol Sci. 2019;20(23). doi: 10.3390/ijms20236008. [PubMed: 31795299]. [PubMed Central: PMC6929211]

21. Chen J, Caspi RR, Chong WP. IL-20 receptor cytokines in autoimmune diseases. J Leukoc Biol. 2018;104(5):953-9. doi: 10.1002/JLB.MR1117-471R. [PubMed: 30260500]. [PubMed Central: PMC6298946].

22. Chung H, Arora S, Damji KF, Weis E. Association of pseudoexfoliation syndrome with cardiovascular and cerebrovascular disease: a systematic review and meta-analysis. Can JOphthalmol.2018;53(4):365-72. doi:10.1016/j.jcjo.2017.10.039. [PubMed: 30119791].

23. Kan E, Yilmaz A, Demirag MD, Calik M. Is pseudoexfoliation syndrome a risk factor for cerebro vascular disease? Semin Ophthalmol. 2017;32(2):153-6. doi: 10.3109/08820538.2015.1009559. [PubMed: 25751568].

24. Lienard J, Croxatto A, Aeby S, Jaton K, Posfay-Barbe K, Gervaix A, et al. Development of a new chlamydiales-specific real-time PCR and its application to respiratory clinical samples. J Clin Microbiol. 2011;49(7):2637-42. doi: 10.1128/JCM.00114-11. [PubMed: 21562107]. [PubMed Central: PMC3147821].

25. Chlamydia pneumoniae $16 \mathrm{~S}$ ribosomal RNA sequence. Report No.: 25 Mar 2021. 2021. [PubMed: L06108.1].

26. Chlamydia trachomatis strain Ia/870 16S ribosomal RNA gene, partial sequence. Report No.: 25 Mar 2021. 2021. [PubMed: DQ019305.1].

27. Chlamydia trachomatis strain J/UW-36 $16 \mathrm{~S}$ ribosomal RNA gene, partial sequence. Report No.: 25 Mar 2021. 2021. [PubMed: DQ019306.1].

28. Faris R, Andersen SE, McCullough A, Gourronc F, Klingelhutz AJ, Weber MM. Chlamydia trachomatis serovars drive differential production of proinflammatory cytokines and chemokines depending on the type of cell infected. Front Cell Infect Microbiol. 2019;9:399. doi: 10.3389/fcimb.2019.00399. [PubMed: 32039039]. [PubMed Central: PMC6988789].

29. WHO. Trachoma. 2021, [cited 15 Mar 2021]. Available from: https:// www.who.int/news-room/fact-sheets/detail/trachoma.

30. Balasopoulou A, Kokkinos P, Pagoulatos D, Plotas P, Makri OE, Georgakopoulos $\mathrm{CD}$, et al. Symposium recent advances and challenges in the management of retinoblastoma Globe-saving treatments. BMC Ophthalmol. 2017;17(1):1.

31. El Yazouli L, Seghrouchni F, Hejaji H, Bouazza M, Alami AA, Dakka N et al. Cell-mediated immune response associated with Chlamydia pneumoniae infection in atherosclerotic patients. Microb Pathog. 2020;139:103860. doi: 10.1016/j.micpath.2019.103860. [PubMed: 31707079].

32. Yuki K, Kimura I, Shiba D, Imamura Y, Tsubota K. Elevated serum immunoglobulin $G$ titers against Chlamydia pneumoniae in pri- mary open-angle glaucoma patients without systemic disease.J Glaucoma. 2010;19(8):535-9. doi: 10.1097/IJG.0b013e3181ca7868. [PubMed: 20164795].

33. Miao G, Zhao X, Wang B, Zhang L, Wang G, Zheng N, et al. TLR2/CXCR4 coassociation facilitates Chlamydia pneumoniae infection-induced atherosclerosis. Am J Physiol Heart Circ Physiol. 2020;318(6):H1420-35. doi: 10.1152/ajpheart.00011.2020. [PubMed: 32330088].

34. Duncan SA, Sahu R, Dixit S, Singh SR, Dennis VA. Suppressors of cytokine signaling (SOCS) 1 and SOCS3 proteins are mediators of interleukin-10 modulation of inflammatory responses induced by Chlamydia muridarum and Its major outer membrane protein (MOMP) in mouse j774 macrophages. Mediators Inflamm. 2020;2020:7461742. doi: 10.1155/2020/7461742. [PubMed: 32684836]. [PubMed Central: PMC7333066].

35. Leinonen M. Chlamydia pneumoniae and other risk factors for atherosclerosis. J Infect Dis. 2000;181 Suppl 3:S414-6. doi: 10.1086/315624. [PubMed: 10839726].

36. Sarenac Vulovic TS, Pavlovic SM, Zdravkovic NS. Proinflammatory cytokines induce XFG development. Ocul Immunol Inflamm. 2016;24(6):671-7. doi: 10.3109/09273948.2015.1063672. [PubMed: 26651123].

37. Takai Y, Tanito M, Ohira A. Multiplex cytokine analysis of aqueous humor in eyes with primary open-angle glaucoma, exfoliation glaucoma, and cataract. Invest Ophthalmol Vis Sci. 2012;53(1):241-7. doi: 10.1167/iovs.11-8434. [PubMed: 22159018].

38. Zenkel M, Lewczuk P, Junemann A, Kruse FE, Naumann GO, SchlotzerSchrehardt U. Proinflammatory cytokines are involved in the initiation of the abnormal matrix process in pseudoexfoliation syndrome/glaucoma. Am J Pathol. 2010;176(6):2868-79. doi: 10.2353/ajpath.2010.090914. [PubMed: 20395431]. [PubMed Central: PMC2877848].

39. Wang X, Wong K, Ouyang W, Rutz S. Targeting IL-10 family cytokines for the treatment of human diseases. Cold Spring Harb Perspect Biol. 2019;11(2). doi: 10.1101/cshperspect.a028548. [PubMed: 29038121]. [PubMed Central: PMC6360861].

40. Ouyang W, Rutz S, Crellin NK, Valdez PA, Hymowitz SG. Regulation and functions of the IL-10 family of cytokines in inflammation and disease. Annu Rev Immunol. 2011;29:71-109. doi: 10.1146/annurevimmunol-031210-101312. [PubMed: 21166540].

41. Zhang W, Magadi S, Li Z, Smith CW, Burns AR. IL-20 promotes epithelial healing of the injured mouse cornea. Exp Eye Res. 2017;154:22-9. doi: 10.1016/j.exer.2016.11.006. [PubMed: 27818315]. [PubMed Central: PMC5359042]. 